\title{
Characteristics of Campylobacter Gastroenteritis Outbreaks in Australia, 2001 to 2016
}

\author{
Cameron R.M. Moffatt, ${ }^{1}$ Emily Fearnley, ${ }^{1,2}$ Robert Bell,, ${ }^{3}$ Rose Wright, ${ }^{4}$ Joy Gregory, ${ }^{5}$ \\ Timothy Sloan-Gardner, ${ }^{6}$ Martyn Kirk, ${ }^{1}$ and Russell Stafford ${ }^{3}$
}

\begin{abstract}
Campylobacter spp. are a globally important cause of bacterial gastroenteritis, with Australia experiencing higher rates of illness than many comparable high-income countries. Despite the high disease incidence, outbreaks of campylobacteriosis in Australia are infrequently detected and reported. We examined the epidemiology of Campylobacter outbreaks in Australia, with particular emphasis on assessing transmission routes and evidence as reported during public health investigations. A national register of enteric and foodborne disease outbreaks was used to summarize data on all Campylobacter outbreaks reported in Australia between 2001 and 2016. Outbreak data were reviewed and analyzed for trends over time. Additional information was sought from state and territory epidemiologists, to validate transmission routes. A total of 84 Campylobacter outbreaks were reported, with $51(61 \%)$ being classified as foodborne. Specific food vehicles were identified for $33(65 \%)$ outbreaks, with $28(85 \%)$ implicating chicken or chicken-containing dishes. Although no increase in the proportion of foodborne Campylobacter outbreaks was observed, examination of specific food vehicles demonstrated a significant increase in outbreaks because of poultry-liver containing foods $(p=0.04)$. One quarter of all 1042 outbreak-associated cases occurred in aged-care facilities (ACFs), including 17 associated hospitalizations and three deaths. After review of evidence data, 23 outbreaks (27\%) were determined to have an unknown route of transmission, including 10 (43\%) outbreaks occurring in ACFs. Campylobacter spp. remain a less commonly reported cause of gastroenteritis outbreaks in Australia. Although many reported outbreaks can be linked to foodborne transmission, over a quarter were unable to identify either a food vehicle or transmission source, particularly for outbreaks occurring in aged care. Increased efforts to improve evidence collection and understanding of transmission dynamics for outbreaks of campylobacteriosis, particularly in aged care, are required.
\end{abstract}

Keywords: Campylobacter, disease outbreaks, foodborne diseases, waterborne diseases, zoonoses, homes for the aged

\section{Introduction}

C AMPYLOBACTER SPP. ARE an important cause of gastroenteritis and responsible for an annual global estimate of 96 million (95\% UI 52-177 million) cases of foodborne infection (Kirk et al., 2015). Although campylobacteriosis most commonly presents as a self-limiting enterocolitis, extraintestinal infections (e.g., bacteremia) and postinfectious complications such as Guillain-Barre syndrome, reactive arthritis, and irritable bowel syndrome may also occur (Fitzgerald, 2015).

Poultry, particularly broilers, are recognized as the primary reservoir for foodborne infections, with ruminants such as cattle and sheep and monogastric species such as pigs also being important carriers (Kaakoush et al., 2015). In addition to illness acquired through undercooked or crosscontaminated foods of animal origin, campylobacteriosis

\footnotetext{
${ }^{1}$ National Centre for Epidemiology and Population Health, Research School of Population Health, Australian National University, Canberra, Australia.

${ }^{2}$ South Australian Department for Health and Wellbeing, OzFoodNet, Adelaide, Australia.

${ }^{3}$ OzFoodNet, Communicable Diseases Branch, Queensland Health, Brisbane, Australia.

${ }_{5}^{4}$ Australian Government Department of Health, OzFoodNet, Office of Health Protection, Canberra, Australia.

${ }^{5}$ Department of Health and Human Services, OzFoodNet, Communicable Disease Epidemiology and Surveillance, Melbourne, Australia.

${ }^{6}$ OzFoodNet, Communicable Disease Control, Health Protection Service, Australian Capital Territory Health Directorate, Canberra, Australia.
} 
may also result from animal interactions, ingestion of unpasteurized milk, contaminated water, or less commonly through person-to-person contact (Kaakoush et al., 2015).

In high-income settings like the European Union (EU) and England, where foodborne disease surveillance is well established, Campylobacter spp. are the most commonly reported enteric pathogens (European Food Safety Authority [EFSA], 2017; Nichols et al., 2012). During 2016, 246,307 cases were reported among EU member states (notification rate 66.3 per 100,000 population) and 52,381 cases were reported in England and Wales (notification rate 89.7 per 100,000) (European Food Safety Authority [EFSA], 2017; Gastrointestinal Infections Department, 2018). Similarly in Australia, disease incidence remains high (Moffatt et al., 2017a), with 24,239 confirmed cases reported in 2016, a national notification rate of 100.2 per 100,000 (Australian Government Department of Health, 2019).

Despite the high incidence and case numbers, Campylobacter outbreaks remain less frequently reported in Australia (Unicomb et al., 2009; Moffatt et al., 2017a). This contrasts markedly with Salmonella enterica, another enteric pathogen of public health significance, responsible for $\sim 1000$ outbreaks in Australia between 2001 and 2016 (Ford et al., 2018). Nevertheless, outbreak data contribute to our understanding of Campylobacter's epidemiology, showing food vehicles such as poultry-liver pâté have become an established cause of outbreaks internationally (Little et al., 2010; Lanier et al., 2018).

Using national outbreak and disease notifications data, we examined Campylobacter outbreaks in Australia, expanding upon a previous assessment for the period 2001-2006 (Unicomb et al., 2009). The aim of this study was to provide a contemporary understanding of the epidemiology of campylobacteriosis outbreaks in Australia, with particular focus on transmission routes and evidence levels obtained during investigations.

\section{Materials and Methods}

All Australian states and territories have public health legislation mandating the reporting of Campylobacter infections and suspected outbreaks of food or waterborne gastroenteritis (OzFoodNet Working Group, 2018). However, campylobacteriosis only became notifiable in the state of New South Wales (NSW) in 2017, falling outside of study parameters. OzFoodNet, a national network performing surveillance and investigation of enteric and foodborne disease in Australia, maintains a National Outbreak Register (hereafter called the Register) with all states and territories contributing outbreak data (Kirk et al., 2008). Two states, NSW and Victoria, use local alternatives to the Register for recording details of nonfoodborne Campylobacter outbreaks.

Data were extracted from the Register providing details of Campylobacter outbreaks between 2001 and 2016. Information included nominated transmission routes, settings where outbreaks occurred, settings where food was prepared (if applicable), dates of outbreak notifications, implicated Campylobacter species (if known), numbers of persons exposed, numbers of cases, hospitalizations and deaths, types of epidemiological investigation (case-control study, analytical

Table 1. Definitions for Outbreaks of Campylobacteriosis by Transmission Route

Outbreak

transmission type

Definition

Foodborne outbreak

An event where $\geq 2$ persons are diagnosed with laboratory-confirmed campylobacteriosis after consuming a common food or meal, and where analytical epidemiology or food microbiology implicates the food or meal as the source of illness

Suspected foodborne outbreak

An event where $\geq 2$ persons are diagnosed with laboratory-confirmed campylobacteriosis after consuming a common food or meal, and where compelling descriptive evidence from an epidemiological investigation and/or food safety inspection implicates the food or meal as the source of illness

Waterborne outbreak

An event where $\geq 2$ persons are diagnosed with laboratory-confirmed campylobacteriosis after exposure to a common water source (either drinking or recreational) and where analytical epidemiology or water testing implicates water as the source of illness

Suspected waterborne outbreak

An event where $\geq 2$ persons are diagnosed with laboratory-confirmed campylobacteriosis after exposure to a common water source (either drinking or recreational) and where compelling descriptive evidence from an epidemiological and/or environmental investigation implicates water as the source of illness

Person to person An event where $\geq 2$ persons are diagnosed with laboratory-confirmed campylobacteriosis after outbreak exposure to a person or group of persons known or suspected to be infectious, or an environment where an infected person has been known to have contaminated and where illness onset dates suggest ongoing transmission

Animal to person outbreak

An event where $\geq 2$ persons are diagnosed with laboratory-confirmed campylobacteriosis after animal exposure and compelling descriptive epidemiological or environmental evidence and/or laboratory evidence implicates the animal as the source of illness

Unknown

An event where $\geq 2$ persons are diagnosed with laboratory-confirmed campylobacteriosis after some common exposure but where a mode of transmission is unable to be determined

Unknown (revised)

An event where $\geq 2$ persons are diagnosed with laboratory-confirmed Campylobacter infection after a common exposure but where a recognized mode of transmission is unable to be determined via either outbreak register detail and/or local review by a state or territory epidemiologist 
Table 2. Characteristics of Campylobacter Outbreaks by Transmission Routes, Australia 2001 to 2016

\begin{tabular}{|c|c|c|c|c|c|}
\hline Transmission route & $\begin{array}{c}\text { No. outbreaks } \\
\text { (\% by route) }\end{array}$ & $\begin{array}{c}\text { No. cases } \\
(\% \text { lab-confirmed })\end{array}$ & $\begin{array}{l}\text { Median } \\
\text { no. cases (min. } \\
\text { to max.) }\end{array}$ & $\begin{array}{c}\text { No. hospitalizations } \\
\text { (\% cases) }\end{array}$ & No. deaths \\
\hline Foodborne & $51(60.7)$ & $723(30.7)$ & $10(2$ to 63$)$ & $51(7.1)$ & 2 \\
\hline Waterborne & $5(6.0)$ & $104(30.8)$ & 22 (7 to 46$)$ & $5(4.8)$ & 0 \\
\hline Animal to person & $2(2.4)$ & $16(37.5)$ & $8(6$ to 10$)$ & $1(6.3)$ & 1 \\
\hline Person to person & $3(3.6)$ & $16(81.3)$ & $4(3$ to 9$)$ & $3(18.8)$ & 0 \\
\hline Unknown & $23(27.4)$ & $183(48.1)$ & 6 (3 to 9$)$ & $12(6.6)$ & 0 \\
\hline Total & $84(100)$ & $1042(34.6)$ & $7(2$ to 63$)$ & $72(6.9)$ & 3 \\
\hline
\end{tabular}

cohort study, or case series), and levels of evidence obtained. Outbreak definitions were adapted from the Register's Data Dictionary (Version 1.5, December 2017) to describe outbreaks occurring through specific transmission routes (Table 1). Nominated food vehicles were grouped under the following categories: poultry-liver dishes; chicken kebabs; other chicken/chicken-containing dishes; chicken unspecified; other food vehicle; other poultry-meat; unpasteurized milk; and unknown food vehicle. For this dataset the term poultry is used in reference to domestic fowls, with poultryliver referring to either chicken or duck and poultry-meat used in reference to duck alone. Other chicken/chickencontaining dishes describe food vehicles where chicken has been identified as the ingredient suspected of being contaminated (e.g., pasta dishes or dumplings). To examine trends in foodborne Campylobacter outbreaks we obtained annual totals for foodborne outbreaks reported between 2001 and 2016. Annual campylobacteriosis notification rates per 100,000 population were obtained from the National Notifiable Diseases Surveillance System (Australian Government Department of Health, 2019) for comparison against annual outbreak counts.

To provide greater etiologic certainty, outbreaks with a single laboratory-confirmed Campylobacter case or where additional enteric pathogens were detected were excluded. Also excluded were Campylobacter clusters. A cluster was defined as an increase in infections that are epidemiologically related in time, place, or person where investigations were unable to implicate a common mode of transmission or source of infection responsible for that increase (OzFoodNet Working Group, 2018).

Outbreak details were forwarded to state and territory foodborne disease epidemiologists, including queries regarding missing or potentially erroneous data. Epidemiologists were also asked to review local records to identify other outbreaks not identified in the Register, with this approach identifying one additional outbreak. We obtained separate details for nonfoodborne Campylobacter outbreaks from NSW and Victoria as both use local databases to record nonfoodborne outbreaks.

Each outbreak was assigned an evidence level based on published indicators of evidentiary strength for foodborne disease outbreaks (Moffatt et al., 2016b). Options for evidence included the following: descriptive epidemiological evidence (hereafter called descriptive); statistical; laboratory and descriptive; descriptive and trace-back; laboratory and statistical; laboratory and trace-back; statistical and traceback; and trace-back. For nonfoodborne outbreaks, options referencing trace-back were not considered. Evidence levels were not assigned for outbreaks where the transmission route was unknown. If our reassessment of transmission differed to the original data, we asked state and territory epidemiologists to examine local records to assist with validation. If no compelling detail was available, our reassessment was adopted.

Data describing Campylobacter isolation in food and environmental samples were assessed and included detection in the following: unopened epidemiologically implicated food; opened epidemiologically implicated food; food consumed without epidemiological implication; other microbiological evidence; isolation from premises and equipment; no identification in laboratory samples; or no samples taken. A descriptive assessment of factors contributing to pathogen contamination and survival was also undertaken but excluded growth factors because of data paucity and a reduced likelihood for Campylobacter spp. to proliferate on foods (Park, 2002). Contributing factors to contamination of food or water included ingestion of contaminated raw product intended to be cooked, cross-contamination from raw ingredients, food handler contamination, storage in contaminated environment, ingestion of contaminated raw product intended to be consumed raw, or unknown. Contributing factors related to survival included insufficient time/temperature during cooking or reheating, inadequate disinfection, inadequate acidification, inadequate thawing and cooking, other process failure, or unknown. Evidence levels for contributing factors included assumed or suspected, confirmed through observation during inspection, confirmed verbally during inspection, confirmed with measured evidence, or unknown.

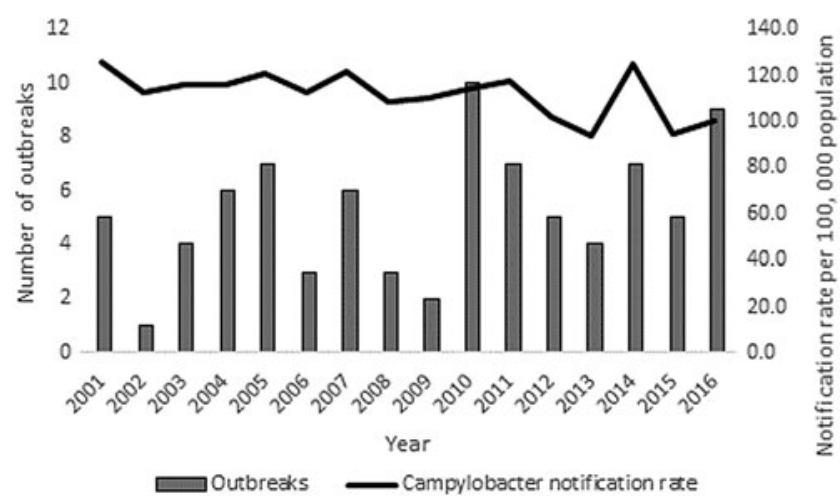

FIG. 1. Annual Campylobacter notification rates and outbreak counts, Australia, 2001-2016. 
Data were analyzed using Microsoft Excel (Microsoft, 2013; Microsoft, Redmond, WA) and Stata SE version 13 (StataCorp, 2013; Stata Statistical Software: Release 13; StataCorp LP, College Station, TX). We used descriptive statistics, including chi-square tests for homogeneity and trend, to assess changes in the proportions of foodborne outbreaks reported over the study period. Examination of aged-care outbreaks used median tests to compare cases, hospitalizations, and outbreak duration between outbreaks classified with foodborne versus unknown transmission routes. The probability value for significance testing was $\leq 0.05$. Ethics approval was obtained from the Australian National University's Human Research Ethics Committee (Protocol: 2018/531).

\section{Results}

Between 2001 and 2016 we identified 84 Campylobacter outbreaks in Australia. Details on transmission route and associated disease burden are given in Table 2, with foodborne or suspected foodborne transmission (hereafter described as foodborne transmission or foodborne outbreaks) accounting for $61 \%$ (51/84) of outbreaks. Outbreaks were reported by all states and territories with the more populous states having higher outbreak counts (data not shown). Speciation detail was available for $44 \%$ (37/84) of outbreaks, with Campylobacter jejuni the dominant etiologic agent (35/ 37). Two outbreaks comprised mixed Campylobacter coli and $C$. jejuni infections. During this time annual notification rates for campylobacteriosis remained high, averaging $>110$ cases per 100,000 population. Conversely, the reported outbreak counts were small (median 5, min. 1 to max. 10), and varied between years (Fig. 1).

Characteristics of foodborne outbreaks by setting are given in Table 3. Among foodborne outbreaks, 39\% (20/51) occurred in the setting of restaurants/cafes, followed by $16 \%$ $(8 / 51)$ in ACFs, and 10\% (5/51) in "other" settings, with the latter including take-away food businesses (small-scale food venues providing made to order foods collected and eaten elsewhere by consumers). When compared with other transmission routes, associations with foodborne transmission were observed in restaurants $\left(\chi^{2}=16.99 p<0.001\right)$ and agedcare facilities (ACFs) $\left(\chi^{2}=7.41 p=0.006\right)$. Implicated foods were mostly prepared within the setting of the outbreak; however, five outbreaks involved foods prepared by commercial caterers, whereas molecular epidemiology linked another community outbreak to commercially produced chicken meat. Food vehicles were identified for 65\% (33/51) of outbreaks, with poultry meat or offal implicated in $85 \%$ (28/33). Among these poultry-related food vehicles, liver dishes (e.g., pâté) comprised 39\% (11/28), followed by other chicken-containing dishes $(33 \%, 8 / 28)$, chicken kebabs $(18 \%, 5 / 28)$, chicken unspecified $(11 \%, 3 / 28)$, and duck (4\%, 1/28). During the study period, OzFoodNet reported 2097 foodborne outbreaks (personal communication Rose Wright, February 13, 2019). After revising transmission route data, we determined that Campylobacter spp. accounted for $2.4 \%$ (51/2083) of foodborne outbreaks between 2001 and 2016. Although no significant trend in foodborne outbreaks caused by Campylobacter spp. was shown, we did observe a significant increase in the proportion of foodborne Campylobacter outbreaks linked to poultry-liver $\left(\chi^{2}=4.22\right.$. $\left.p=0.04\right)$. Among poultry-liver outbreaks, $73 \%(8 / 11)$ involved restaurants, $18 \%$ (2/11) involved commercial catering, whereas one outbreak involved pâté prepared in a private residence.

Nonfoodborne outbreak characteristics are given in Table 2. Before review, $11 \%$ (9/84) of Campylobacter outbreaks had an unknown transmission route. This increased to $27 \%(23 / 84)$ after we identified 14 outbreaks recorded as being foodborne but lacking the necessary evidence to meet the foodborne outbreaks definitions as given in Table 1 . Among the most frequently identified settings for outbreaks with an unknown source were ACFs $(n=10)$, military facilities $(n=4)$, and private residences $(n=4)$. Among

Table 3. Characteristics of Foodborne Campylobacter Outbreaks by Setting, Australia 2001-2016

\begin{tabular}{|c|c|c|c|c|c|}
\hline \multirow[b]{2}{*}{ Outbreak setting } & \multirow[b]{2}{*}{$\begin{array}{l}\text { No. outbreaks } \\
\text { (all routes) }\end{array}$} & \multicolumn{4}{|c|}{ Foodborne outbreaks } \\
\hline & & $\begin{array}{l}\text { No. outbreaks } \\
\text { (\% foodborne) }\end{array}$ & $\begin{array}{c}\text { No. cases } \\
\text { (\% lab-confirmed })\end{array}$ & $\begin{array}{l}\text { Median no. } \\
\text { cases (min. } \\
\text { to max.) }\end{array}$ & $\begin{array}{c}\text { No. hospitalizations } \\
\text { (No. deaths) }\end{array}$ \\
\hline Restaurants & 20 & $20(100.0)$ & $228(28.9)$ & $10(2$ to 35$)$ & 13 \\
\hline Aged care & 22 & $8(36.4)$ & $143(28.0)$ & 14.5 (3 to 49$)$ & $10(2)$ \\
\hline Other settings ${ }^{\mathrm{a}}$ & 5 & $5(100.0)$ & $85(32.9)$ & $5(2$ to 63$)$ & 1 \\
\hline Camps & 7 & $3(42.9)$ & 43 (14.0) & 14 (6 to 23$)$ & 0 \\
\hline Private residences & 9 & $3(33.3)$ & $16(50.0)$ & $5(4$ to 7$)$ & 0 \\
\hline Schools & 2 & $2(100.0)$ & $42(38.1)$ & 21 (6 to 36$)$ & 1 \\
\hline Other institutions & 3 & $2(66.7)$ & $61(14.8)$ & 30.5 (5 to 56$)$ & 1 \\
\hline Picnics & 2 & $2(100.0)$ & $8(87.5)$ & 4 & 0 \\
\hline Community & 2 & $2(100.0)$ & $27(100.0)$ & 13.5 (3 to 24$)$ & 4 \\
\hline Military facility & 5 & $1(20.0)$ & $19(15.8)$ & 19 & 19 \\
\hline Commercial caterer & 1 & $1(100.0)$ & $11(54.5)$ & 11 & 1 \\
\hline Function & 1 & $1(100.0)$ & 31 (12.9) & 31 & 1 \\
\hline Workplace & 1 & $1(100.0)$ & $9(22.2)$ & 9 & 0 \\
\hline Totals & $81^{\mathrm{b}}$ & 51 & $723(30.7)$ & 10 (2 to 63$)$ & $51(2)$ \\
\hline
\end{tabular}

${ }^{\mathrm{a} I n c l u d e s ~ t h r e e ~ t a k e a w a y ~ f o o d ~ e s t a b l i s h m e n t s, ~ a ~ s p o r t i n g ~ c l u b, ~ a n d ~ a ~ d a i r y ~ f a r m . ~}$

${ }^{\mathrm{b}}$ Eighty-four outbreaks identified in total. Three nonfoodborne outbreaks occurred in settings not described above, that is, a child care center, a hospital, and an unknown setting. 
Table 4. Characteristics of CampYlobacter Outbreaks Reported in Australian Aged-Care FACILITIES, 2001-2016

\begin{tabular}{|c|c|c|c|c|c|c|}
\hline $\begin{array}{l}\text { Transmission } \\
\text { route }\end{array}$ & $\begin{array}{c}\text { No. } \\
\text { outbreaks (\%) }\end{array}$ & $\begin{array}{c}\text { No. } \\
\text { cases }(\%)\end{array}$ & $\begin{array}{c}\text { No. } \\
\text { lab-confirmed } \\
\text { cases (\%) }\end{array}$ & $\begin{array}{c}\text { Median } \\
\text { no. cases } \\
\text { (min. to max.) }\end{array}$ & $\begin{array}{c}\text { No. } \\
\text { hospitalizations } \\
\text { (total deaths) }\end{array}$ & $\begin{array}{c}\text { Median outbreak } \\
\text { duration in hours } \\
\text { (min. to max.) }\end{array}$ \\
\hline Unknown & $10(45.4)$ & $82(32.5)$ & $35(39.8)$ & 6.5 (3 to 15$)$ & $3(0)$ & $72(24 \text { to } 120)^{\mathrm{a} 6}$ \\
\hline Foodborne & $8(36.6)$ & $143(56.7)$ & $40(45.5)$ & 14.5 (3 to 49$)$ & $10(2)$ & $72(24 \text { to } 168)^{a 7}$ \\
\hline Animal to person & $2(9.0)$ & $16(6.3)$ & $66.8)$ & $8(6$ to 10$)$ & $1(1)$ & $144^{\mathrm{a} 1}$ \\
\hline Waterborne & $1(4.5)$ & $7(2.8)$ & $3(3.4)$ & $7(-)$ & $0(0)$ & $120^{\mathrm{a} 1}$ \\
\hline Person to person & $1(4.5)$ & $4(1.6)$ & $4(4.5)$ & $4(-)$ & $3(0)$ & No data \\
\hline Totals & $22(100.0)$ & $252(100.0)$ & $88(100.0$ & 8.5 (3 to 49$)$ & $17(3)$ & $96(24 \text { to } 168)^{\mathrm{a} 15}$ \\
\hline
\end{tabular}

${ }^{\mathrm{a}}$ Number of outbreaks with data.

waterborne outbreaks $(n=5)$, three occurred in camps, with single outbreaks in a child care center and an ACF. Rainwater tanks were identified as the source for four outbreaks, with a water bore the source for the fifth. Outbreaks involving person-to-person transmission were reported in an ACF, child care center, and private residence, whereas two outbreaks in a single ACF were linked to zoonotic transfer (Moffatt et al., 2014).

Outbreaks were reported most frequently in ACFs, with $26 \%(22 / 84)$ occurring in this setting. Transmission source and associated disease burden for ACF outbreaks are given in Table 4. Among ACF outbreaks, 36\% (8/22) involved foodborne transmission, whereas $45 \%(10 / 22)$ had an unknown transmission source. Foodborne outbreaks in ACFs were larger (median 14.5 cases) than those with an unknown transmission route (median 6.5 cases), whereas hospitalization frequency was significantly higher $\left(\chi^{2}=4.74 . p=0.03\right)$. Among foodborne outbreaks in ACFs, three-quarters $(6 / 8)$ did not identify a food vehicle.

Examination of evidence levels obtained among the $73 \%$ $(61 / 84)$ of outbreaks where a transmission route was determined showed $49 \%(30 / 61)$ utilized descriptive epidemiological evidence, $21 \%$ (13/61) statistical evidence, whereas $13 \%(8 / 61)$ used descriptive and statistical evidence in conjunction with microbiological findings. A further 10 outbreaks, including nine reported as foodborne, relied upon limited descriptive evidence, most often a common meal or restaurant exposure among cases but without a specific food vehicle being identified.

Data on food and environmental sampling conducted during investigations was available for 81 outbreaks, with $73 \%(59 / 81)$ of these investigations having no food or other samples collected for microbiological testing. For outbreaks involving sampling, 59\% (19/32) yielded negative results, whereas $25 \%$ produced supportive evidence only (e.g., the presence of fecal indicator bacteria in a water sample). Campylobacter spp. were detected only four times in epidemiologically implicated foods, comprising chicken-liver pâté, duck livers, chicken wontons, and retail chicken meat. Campylobacter spp. was also recovered once from a rainwater tank supplying drinking water.

Investigators identified 57\% (29/51) of foodborne outbreaks had one or more factors associated with pathogen contamination, whereas $47 \%$ (24/51) detailed factors relating to pathogen survival. Issues most frequently recorded were the ingestion of contaminated raw products intended to be cooked $(n=17)$ and cross-contamination from raw ingredi- ents $(n=14)$, whereas for survival factors, insufficient temperature or cooking time was identified in $92 \%(22 / 24)$ of outbreaks. Most evidence used in determining contamination $(83 \%, 24 / 29)$ and survival factors $(71 \%, 17 / 24)$ were based on investigator assumption, as opposed to direct observation or measurement.

\section{Discussion}

Our study suggests that Campylobacter gastroenteritis outbreaks occur infrequently in Australia. Among outbreaks with recognized transmission sources, most were foodborne, with chicken or chicken-containing dishes the most commonly identified food vehicles. Although no rise in outbreaks over time was observed we did detect an increase in foodborne outbreaks involving poultry-liver. Other transmission sources seem to play a limited role in causing outbreaks in Australia. ACFs were the most frequently reported outbreak setting, whereas foodborne transmission occurred mostly in restaurants. A number of ACF outbreaks were reclassified to having an unknown transmission source, highlighting the challenges when gathering evidence to support inferences of foodborne transmission in that setting.

Despite robust food safety standards, Australia like many high-income countries, experiences significant disease burden because of Campylobacter spp. (Havelaar et al., 2015; Moffatt et al., 2017a). Despite this burden our results show a median of five outbreaks per year, with no increase in foodborne Campylobacter outbreaks as a proportion of all foodborne outbreaks over time. This infrequency of outbreaks relative to disease burden may be partly explained by Campylobacter's innate physiology. Although invariably present on foods like raw poultry, the organism's fastidious growth requirements, coupled with sensitivity to environmental stressors limit its capacity to proliferate on food and survive cooking and freezing (Park, 2002). Similarly, international reporting shows Campylobacter contributes only a small proportion of the foodborne outbreak burden, although some increases are being reported. Between 2004 and 2008, the United States observed a two-fold increase (Geissler et al., 2017), whereas in England the proportion of Campylobacter outbreaks increased significantly between 1992 and 2008, despite a decline in overall foodborne outbreaks (Gormley et al., 2011). Such apparent differences with Australia could be attributed to variations in risk factors, transmission routes, surveillance and notification practices, or food regulation requirements. 
Campylobacteriosis is recognized as a predominantly foodborne illness, with poultry being the major reservoir and the consumption and handling of poultry meat major risk factors (Skarp et al., 2016). Globally, meat consumption has risen markedly over the past 20 years, driven largely by increases in poultry consumption, with Australians consuming $>40 \mathrm{~kg}$ of poultry-meat per capita each year (Australian Bureau of Agricultural and Resource Economics and Sciences (ABARES) 2019). Consistent with this, our findings show a majority of outbreaks with a known transmission route were recorded as being foodborne, with chicken or poultrycontaining meals the primary food vehicles. Although this study did not observe any increase in foodborne Campylobacter outbreaks per se, it did identify a significant increase in outbreaks linked to food vehicles such as pâté (Parry et al., 2012; Moffatt et al., 2016a), mirroring international outbreak reports implicating poultry-liver dishes (Little et al., 2010; Lanier et al., 2018). The ability of Campylobacter spp. to penetrate poultry livers through the hepatobiliary ducts (Whyte et al., 2006), coupled with changes in eating habits and cooking practices (Jones et al., 2016), provide a strong rationale for this observed increase.

Another food vehicle group responsible for outbreaks are unpasteurized dairy products. In the United States, these account for $69 \%$ of foodborne Campylobacter outbreaks (Geissler et al., 2017), whereas we identified a single outbreak linked to unpasteurized milk, representing $<2 \%$ of foodborne Campylobacter outbreaks. This discrepancy is explained by regulatory differences where in the United States, despite a federal ban on interstate commercial sales, numerous states permit sale of unpasteurized products within the state in which they were produced (Langer et al., 2012). In contrast, Australia legally requires all milk and dairy products undergo pasteurization (or an equivalent process) to eliminate pathogenic microorganisms (Food Standards Australia and New Zealand (FSANZ), 2009), thus largely avoiding the issue.

Water sources are another recognized cause of Campylobacter outbreaks, with numerous large-scale events involving ground and surface water being reported throughout Europe, North America, and New Zealand (Moreira et al., 2017). Although Australian estimates suggest $11 \%$ of campylobacteriosis cases are linked to water exposure (Gibney et al., 2017), the majority of waterborne outbreaks have involved recreational exposure to Cryptosporidium infections (Dale et al., 2010). Our study identified five waterborne outbreaks linked to drinking water, with four involving rainwater storage tanks and the fifth linked to bore water. Using rainwater tanks for household drinking water is common practice in Australia, particularly in rural and remote areas and the microbiological quality of such water is recognized as being less reliable (Environmental Health Committee (enHealth), 2011). Overall outbreak risk because of drinking water is probably low as a majority of Australians can access highquality, regulated mains water; however, private supplies, particularly those utilizing rainwater storage tanks, carry some risk.

This study identified two outbreaks in an ACF involving probable zoonotic transmission through a puppy, highlighting a potentially emerging area of concern. Carriage and excretion of Campylobacter spp. in puppies and adolescent dogs is well-recognized (Hald et al., 2004), whereas associ- ations between human illness and puppy ownership have been demonstrated (Stafford et al., 2007; Mughini Gras et al., 2013). Although cognizant of the benefits of companion and therapy animals, the presence of puppies in such settings raises human health concerns (Moffatt et al., 2014). Given our study has reported a number of ACF outbreaks without identified transmission sources we would encourage active efforts to exclude potential zoonotic transfer by scrutinizing age and species detail for all visiting or resident animals.

Our study shows ACFs to be the most common setting for Campylobacter outbreaks in Australia. Between 2001 and 2006, 18\% (6/33) of outbreaks occurred in ACFs (Unicomb et al., 2009), increasing to $26 \%$ (22/84) during our review period. Previously, Campylobacter spp. was shown to be the third highest cause of foodborne outbreaks in Australian ACFs (Kirk et al., 2011). This contrasts with U.S. and UK data showing few foodborne aged-care outbreaks are attributed to Campylobacter spp. (Levine et al., 1991; Ryan et al., 1997). Such discrepancies could relate to a reporting bias, with all Australian states and territories requiring ACFs to report gastroenteritis outbreaks. Although this practice is well established in Australia, (Communicable Diseases Network Australia, 2010), potential underreporting of outbreak events should not be dismissed.

Although there are suggestions that higher rates of foodborne outbreak reporting in Australian ACFs may relate to more intensive investigation practices (Kirk et al., 2012), our study shows a high proportion of these outbreaks lack evidence of foodborne transmission. Aged care is a challenging environment for investigations, with attempts to interview residents or retrospectively examine documentation leading to inconclusive findings (Winquist et al., 2001). Our study shows food and environmental samples collected during outbreak investigations rarely result in Campylobacter spp. being detected, with this outcome in contrast to the relative successes achieved with trace-back sampling for $S$. enterica after egg-associated outbreaks in Australia (Moffatt et al., 2017b). Given the proportion of ACF outbreaks with unknown transmission and their differing characteristics with outbreaks we have classified as foodborne, alternative transmission routes require consideration.

The Register has proved an invaluable tool in assisting varied public health and regulatory stakeholders with monitoring disease trends, developing policy and guiding food safety programs (Kirk et al., 2008). We believe our liaison processes with states and territories, particularly requesting reviews of local records, have improved the quality of data presented, while addressing previous concerns regarding weak evidence and poor documentation of Register-derived data (Kirk et al., 2011). A key aim of this study was to assess the quality of evidence used in assigning transmission routes, with the results showing that a number of outbreaks nominally recorded as being foodborne lacked the evidence to meet foodborne outbreak case definitions. Nevertheless differences in surveillance practices may have led to some foodborne outbreaks not being reported, with not all states and territories routinely contributing data to the Register until 2003. In addition, NSW only mandated the requirement to notify campylobacteriosis in 2017.

Public health capacity to routinely investigate even a small proportion of Campylobacter cases is currently constrained, with further difficulties imposed by the absence of a routine 
subtyping system. However, a recent Danish study has shown whole genome sequencing may identify a higher degree of Campylobacter clustering and outbreak occurrence than previously thought (Joensen et al., 2018). This illustrates the potential for this method as the basis for integrated surveillance routinely comparing human-derived isolates against those from established reservoirs. Although this could assist with detection of community-level outbreaks and the identification of sources within the primary production and processing sector, it remains possible that point-source foodborne outbreaks will remain uncommon because of Campylobacter's physiology.

\section{Conclusions}

Campylobacter spp. remain a less commonly identified cause of gastroenteritis outbreaks in Australia. Although most outbreaks can be linked to foodborne transmission, including an increasing number caused by poultry-liver dishes, our study shows that gaps persist in evidence and knowledge around transmission. This is particularly evident for ACFs, where a higher frequency of outbreaks and an increased susceptibility of residents to poor health outcomes, mean efforts to better elucidate outbreak causes should be afforded a high priority.

\section{Acknowledgments}

The authors thank the following OzFoodNet Working Group members who have assisted with this study: Anthony Draper (Northern Territory), Neil Franklin (New South Wales), Kim Lilly (Hunter New England), Tasmania (Michelle Harlock), and Benjamin Witham (Western Australia).

\section{Disclosure Statement}

No competing financial interests exist.

\section{Funding Information}

The OzFoodNet Working Group is funded by the Australian Government Department of Health. C.M. has been supported by a National Health and Medical Research Council Public Health and Health Services Postgraduate Research Scholarship (APP107490). M.K. is supported by a National Health and Medical Research Council Fellowship (APP1145997).

\section{References}

Australian Bureau of Agricultural and Resource Economics and Sciences (ABARES). Agricultural Commodities: March Quarter 2019. Canberra: Commonwealth of Australia, 2019. Australian Government Department of Health. National Notifiable Diseases Surveillance System. Notification Rates (per 100,000 population) 1991 - present, Australia. Canberra, ACT: Australian Government, 2019.

Communicable Diseases Network Australia. Guidelines for the Public Health Management of Gastroenteritis Outbreaks Due to Norovirus or Suspected Viral Agents in Australia. Australian Government Department of Health, (ed.): Commonwealth of Australia, 2010.

Dale K, Kirk M, Sinclair M, Hall R, Leder K. Reported waterborne outbreaks of gastrointestinal disease in Australia are predominantly associated with recreational exposure. Aust $\mathrm{N}$ Z J Public Health 2010;34:527-530.
Environmental Health Committee (enHealth). Guidance on use of rainwater tanks. Australian Government Department of Health, (ed.). Canberra: Commonwealth of Australia, 2011.

European Food Safety Authority (EFSA) and European Centre for Disease Control (ECDC). The European Union summary report on trends and sources of zoonoses, zoonotic agents and food-borne outbreaks in 2016. EFSA J 2017;15:5077.

Fitzgerald C. Campylobacter. Clin Lab Med 2015;35:289-298.

Food Standards Australia and New Zealand (FSANZ). Standard 4.2.4 Primary Production and Processing Standard for Dairy Products (Australia Only). Canberra: FSANZ, 2009.

Ford L, Moffatt CRM, Fearnley E, et al. The Epidemiology of Salmonella enterica Outbreaks in Australia, 2001-2016. Front Sustain Food Syst 2018;2:86.

Gastrointestinal Infections Department. Campylobacter Data 2007 to 2016. Public Health England, 2018.

Geissler AL, Bustos Carrillo F, Swanson K, et al. Increasing Campylobacter Infections, Outbreaks, and Antimicrobial Resistance in the United States, 2004-2012. Clin Infect Dis 2017;65:1624-1631.

Gibney KB, O’Toole J, Sinclair M, Leder K. Burden of Disease Attributed to Waterborne Transmission of Selected Enteric Pathogens, Australia, 2010. Am J Trop Med Hyg 2017;96: 1400-1403.

Gormley FJ, Little CL, Rawal N, Gillespie IA, Lebaigue S, Adak GK. A 17-year review of foodborne outbreaks: Describing the continuing decline in England and Wales (19922008). Epidemiol Infect 2011;139:688-699.

Hald B, Pedersen K, Waino M, Jorgensen JC, Madsen M. Longitudinal study of the excretion patterns of thermophilic Campylobacter spp. in young pet dogs in Denmark. J Clin Microbiol 2004;42:2003-2012.

Havelaar AH, Kirk MD, Torgerson PR, et al. World Health Organization Global Estimates and Regional Comparisons of the Burden of Foodborne Disease in 2010. PLoS Med 2015; 12:e1001923.

Joensen KG, Kuhn KG, Muller L, et al. Whole-genome sequencing of Campylobacter jejuni isolated from Danish routine human stool samples reveals surprising degree of clustering. Clin Microbiol Infect 2018;24:201 e5-e201 e8.

Jones AK, Rigby D, Burton M, et al. Restaurant cooking trends and increased risk for Campylobacter infection. Emerg Infect Dis 2016;22:1208-1215.

Kaakoush NO, Castano-Rodriguez N, Mitchell HM, Man SM. Global epidemiology of Campylobacter infection. Clin Microbiol Rev 2015;28:687-720.

Kirk MD, Gregory J, Lalor K, Hall GV, Becker N. Foodborne and waterborne infections in elderly community and longterm care facility residents, Victoria, Australia. Emerg Infect Dis 2012;18:377-384.

Kirk MD, Lalor K, Raupach J, et al. Food- and waterborne disease outbreaks in Australian long-term care facilities, 2001-2008. Foodborne Pathog Dis 2011;8:133-139.

Kirk MD, McKay I, Hall GV, et al. Food safety: Foodborne disease in Australia: The OzFoodNet experience. Clin Infect Dis 2008;47:392-400.

Kirk MD, Pires S.M, Black RE, et al. World Health Organization Estimates of the Global and Regional Disease Burden of 22 Foodborne Bacterial, Protozoal, and Viral Diseases, 2010: A Data Synthesis. PLoS Med 2015;12:e1001921.

Langer AJ, Ayers T, Grass J, Lynch M, Angulo FJ, Mahon BE. Nonpasteurized dairy products, disease outbreaks, and state laws-United States, 1993-2006. Emerg Infect Dis 2012;18: 385-391. 
Lanier WA, Hale KR, Geissler AL, Dewey-Mattia D. Chicken Liver-Associated Outbreaks of Campylobacteriosis and Salmonellosis, United States, 2000-2016: Identifying Opportunities for Prevention. Foodborne Pathog Dis 2018;15:726-733.

Levine WC, Smart JF, Archer DL, Bean NH, Tauxe RV. Foodborne disease outbreaks in nursing homes, 1975 through 1987. JAMA 1991;266:2105-2109.

Little CL, Gormley FJ, Rawal N, Richardson JF. A recipe for disaster: Outbreaks of campylobacteriosis associated with poultry liver pate in England and Wales. Epidemiol Infect 2010;138:1691-1694.

Moffatt C, Appuhamy R, Andrew W, Wynn S, Roberts J, Kennedy K. An assessment of risk posed by a Campylobacter-positive puppy living in an Australian residential aged-care facility. Western Pac Surveill Response J 2014;5:1-6.

Moffatt CR, Glass K, Stafford R, D'Este C, Kirk MD. The campylobacteriosis conundrum - examining the incidence of infection with Campylobacter sp. in Australia, 1998-2013. Epidemiol Infect 2017a;145:839-847.

Moffatt CR, Greig A, Valcanis M, et al. A large outbreak of Campylobacter jejuni infection in a university college caused by chicken liver pate, Australia, 2013. Epidemiol Infect 2016a;144:2971-2978.

Moffatt CR, Musto J, Pingault N, et al. Salmonella Typhimurium and Outbreaks of Egg-Associated Disease in Australia, 2001 to 2011. Foodborne Pathog Dis 2016b;13:379-385.

Moffatt CRM, Musto J, Pingault N, et al. Recovery of Salmonella enterica from Australian Layer and Processing Environments Following Outbreaks Linked to Eggs. Foodborne Pathog Dis 2017b;14:478-482.

Moreira NA, Bondelind M. Safe drinking water and waterborne outbreaks. J Water Health 2017;15:83-96.

Mughini Gras L, Smid JH, Wagenaar JA, et al. Increased risk for Campylobacter jejuni and C. coli infection of pet origin in dog owners and evidence for genetic association between strains causing infection in humans and their pets. Epidemiol Infect 2013;141:2526-2535.

Nichols GL, Richardson JF, Sheppard SK, Lane C, Sarran C. Campylobacter epidemiology: A descriptive study reviewing 1 million cases in England and Wales between 1989 and 2011. BMJ Open 2012;2: pii: e001179.
OzFoodNet Working Group. Monitoring the incidence and causes of diseases potentially transmitted by food in Australia: Annual report of the OzFoodNet network, 2012. Commun Dis Intell (2018) 2018;42: pii: S22096051(18)00014-3.

Park SF. The physiology of Campylobacter species and its relevance to their role as foodborne pathogens. Int $\mathrm{J}$ Food Microbiol 2002;74:177-188.

Parry A, Fearnley E, Denehy E. 'Surprise': Outbreak of Campylobacter infection associated with chicken liver pate at a surprise birthday party, Adelaide, Australia, 2012. Western Pac Surveill Response J 2012;3:16-19.

Ryan MJ, Wall PG, Adak GK, Evans HS, Cowden JM. Outbreaks of infectious intestinal disease in residential institutions in England and Wales 1992-1994. J Infect 1997;34: 49-54.

Skarp CPA, Hanninen ML, Rautelin HIK. Campylobacteriosis: The role of poultry meat. Clin Microbiol Infect 2016;22:103109.

Stafford RJ, Schluter P, Kirk M, et al. A multi-centre prospective case-control study of campylobacter infection in persons aged 5 years and older in Australia. Epidemiol Infect 2007;135:978-988.

Unicomb LE, Fullerton KE, Kirk MD, Stafford RJ. Outbreaks of campylobacteriosis in Australia, 2001 to 2006. Foodborne Pathog Dis 2009;6:1241-1250.

Whyte R, Hudson JA, and Graham C. Campylobacter in chicken livers and their destruction by pan frying. Lett Appl Microbiol 2006;43:591-595.

Winquist AG, Roome A, Mshar R, Fiorentino T, Mshar P, Hadler J. Outbreak of campylobacteriosis at a senior center. J Am Geriatr Soc 2001;49:304-307.

Address correspondence to: Cameron R.M. Moffatt, MPH, MAppEpid National Centre for Epidemiology and Population Health Research School of Population Health Australian National University Acton 2601 Australia

E-mail: cameron.moffatt@anu.edu.au 